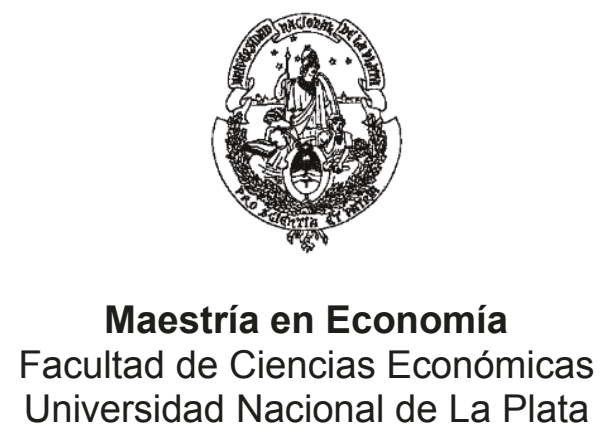

TESIS DE MAESTRIA

ALUMNO

Juan Marcos Pastor

TITULO

Dinámica inflacionaria y persistencia: Argentina 1980-2013

DIRECTOR

Lorena Garegnani

FECHA DE DEFENSA

$28 / 11 / 2014$ 


\title{
Dinámica inflacionaria y persistencia: Argentina 1980-2013
}

\author{
Juan Marcos Pastor ${ }^{1}$ \\ Tesis de Maestría \\ Maestría en Economía \\ Universidad Nacional de La Plata \\ Directora de Tesis: Lorena Garegnani
}

28 de noviembre de 2014

\section{Resumen}

En este trabajo se estudia la dinámica de la inflación agregada, en particular el fenómeno de la persistencia inflacionaria en el período 1980-2013 en Argentina siguiendo dos enfoques: el de series de tiempo univariado y el de modelos de vectores autorregresivos (VAR) para comprender el vínculo de su dinámica con otras variables macro-monetarias de la economía. Utilizando métodos recursivos y test de cambios estructural desarrollados por Bai y Perron identificamos quiebres en la tasa media de inflación que coinciden con cambios en el régimen monetario. En el modelo VAR se encuentra que, si bien la mitad de la variabilidad de la inflación depende de su propia dinámica, los impulsos monetarios la afectan de manera positiva y significativa.

Clasificación JEL: C22, E31, E40, E52

Palabras claves: persistencia inflacionaria, media variable, series de tiempo, modelos VAR

\footnotetext{
${ }^{1}$ Email: juanmarcospastor@gmail.com
} 


\section{Índice}

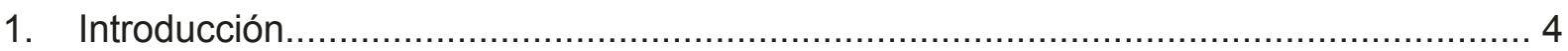

2. Enfoque metodológico y revisión de la literatura existente. ....................................... 5

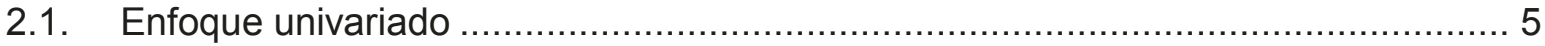

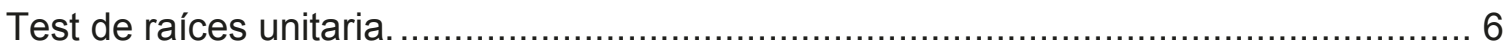

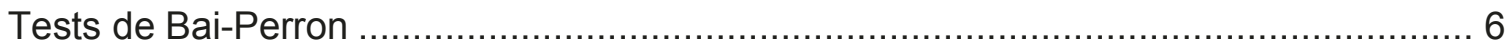

Suma de los coeficientes autoregresivos de la inflación ........................................... 6

2.2. Dinámica inflacionaria. Enfoque Multivariado: VAR ............................................ 7

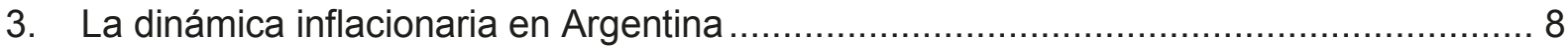

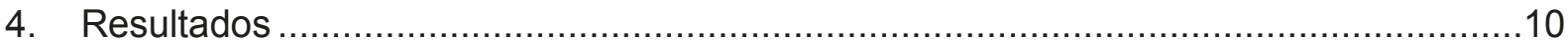

4.1. Enfoque Univariado: Persistencia de la Inflación ...............................................11

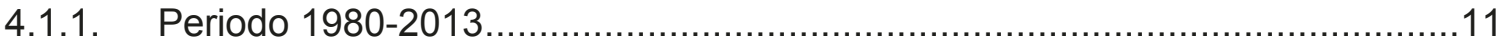

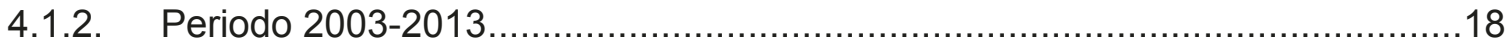

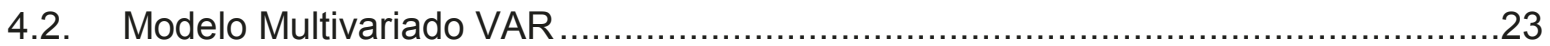

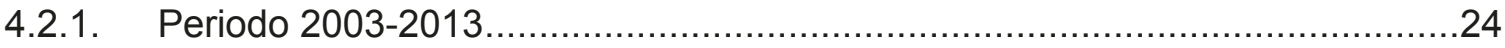

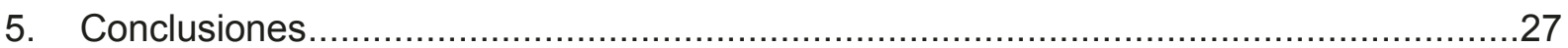

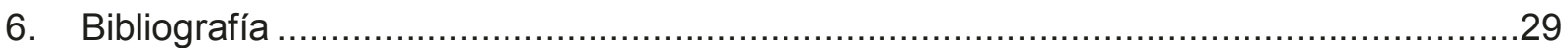

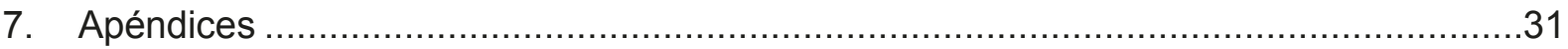

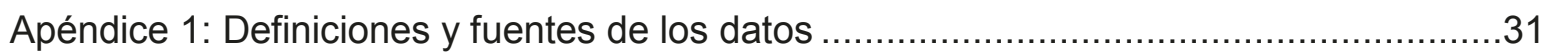

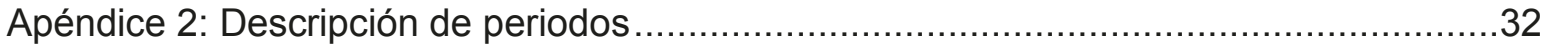

Apéndice 3: Desarrollo de las funciones de impulso-respuesta y la descomposición de

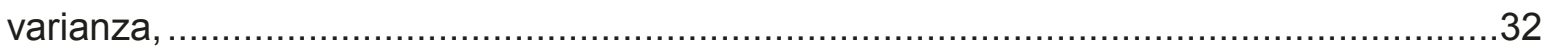

Apéndice 4: Análisis descriptivo y gráficos de variables del sistema VAR .........................34 


\section{Introducción}

La evidencia empírica reciente ha revelado que la persistencia puede no ser una característica intrínseca a la inflación y que la dinámica inflacionaria puede cambiar dependiendo del régimen monetario que prevalezca en la economía. Estos estudios revelan además, la importancia de considerar la posibilidad de que el valor de largo plazo de la inflación experimente quiebres cuando se calculan medidas de persistencia inflacionaria.

Según Fuhrer \& Moore (1995) y Levin \& Piger (2004), un hecho estilizado bastante establecido sobre la dinámica de la inflación ha sido su alta persistencia, esta característica tiene consecuencias muy importantes tanto para la modelación como para el accionar de política monetaria.

A pesar de que los cambios en la inflación tendencial parecen ser empíricamente relevantes para la dinámica inflacionaria, los modelos neokeynesianos utilizados para modelar la política monetaria, al enfocarse en las fluctuaciones de corto plazo de la inflación y el producto, suponen en general una tasa de inflación cero para el estado estacionario, dejando de lado la posibilidad de que la inflación de largo plazo pueda ser positiva y eventualmente variable en el tiempo.

Recientemente Cogley, Primiceri, \& Sargent (2008), Cogley \& Sbordone (2006) y Sbordone (2007) han mostrado para los Estados Unidos que la persistencia de la inflación puede explicarse por la presencia de una inflación de tendencia variable en el tiempo. Al mismo tiempo Benati (2007) y Altissimo, et al. (2006) muestran para un número importante de países que el grado de persistencia de la inflación ha variado en el tiempo y que ha sido más bajo en períodos en los que la política monetaria pudo estabilizar la inflación en niveles reducidos.

A lo largo de las últimas tres décadas, Argentina ha experimentado varios regímenes monetarios y crisis económicas. El supuesto de un valor de largo plazo constante para la tasa de inflación es sin duda implausible para una economía como la Argentina, que experimentó inflación elevada en los 80 s, cuando el ejercicio de la política monetaria estuvo fuertemente condicionado por desequilibrios fiscales persistentes que implicaban una alta dominancia fiscal. Ese régimen de alta inflación derivó hacia fines de la década de los $80 \mathrm{~s}$ en un episodio hiperinflacionario luego del cual se adoptó un régimen monetario de caja de conversión, la Convertibilidad, en el que la dinámica inflacionaria estaba gobernada, en gran medida, por factores externos. La inflación se mantuvo en niveles reducidos durante ese período, que finalizó con el abandono de ese régimen en enero de 2002. Luego de la abrupta devaluación del peso, la inflación se aceleró, alcanzando un valor máximo en abril 
de 2002, para luego retornar a valores más reducidos, aunque superiores a los niveles promedio durante la Convertibilidad. A partir del 2007 la inflación comienza nuevamente a incrementarse debido a un aumento en el gasto público y a un modelo de tipo de cambio real alto. Esto trae como consecuencia un incremento en la emisión monetaria y en el tipo de cambio nominal.

En este trabajo se estudia la dinámica de la inflación agregada, en particular el fenómeno de la persistencia inflacionaria en el período 1980-2013 en Argentina. Si la inflación fuese un proceso fuertemente persistente, los shocks sobre esta variable tardarían mucho tiempo en revertirse y, en este caso, producir una desinflación podría ser un proceso altamente costoso en términos de nivel de actividad. Es por esta razón que luego de estudiar la persistencia de la inflación de manera univariada se procede a introducirla en un contexto multivariado a través de modelos de vectores autorregresivos (VAR) para comprender el vínculo de su dinámica con aquellas variables económicas relevantes en la transmisión de los impulsos nominales a la economía.

El aporte que este trabajo intenta hacer a la literatura sobre dinámica inflacionaria y persistencia en Argentina es, en primer lugar, extender el análisis realizado para Argentina por D'Amato, Garegnani, \& Sotes (2008) y D'Amato \& Garegnani (2013) quienes finalizan su período de análisis en el año 2007. En segundo lugar contribuir a la discusión tan actual sobre los determinantes de la dinámica inflacionaria y de la formación de precios mediante técnicas que permiten conocer su vínculo con otras variables macroeconómicas.

La siguiente sección detalla el marco teórico y la revisión de literatura. La tercera está compuesta por el análisis de los datos y la descripción del periodo. En la cuarta sección se presentan los resultados y en la quinta se concluye.

\section{Enfoque metodológico y revisión de la literatura existente.}

En esta sección se explica la metodología que se va a implementar para abordar el estudio de la dinámica inflacionaria. Se comienza con el estudio de la característica de persistencia inflacionaria, utilizado en el análisis univariado de la inflación; y luego se presenta un breve desarrollo de Sistemas VAR, utilizado en el análisis multivariado para investigar el vínculo de la inflación con otras variables (tasa de interés, tipo de cambio, dinero y $\mathrm{PBI}$ ) relevantes para la transmisión de los impulsos nominales a la economía.

\subsection{Enfoque univariado}

El término persistencia, analizado desde un punto de vista estadístico, es una medida de la velocidad con que una variable retorna a su valor medio. Si este concepto lo incluimos en 
economía monetaria, se refiere a la velocidad con que la inflación $\left(\pi_{t}\right)$ retorna a su valor de equilibrio de largo plazo luego de un shock.

Fuhrer (2009) menciona las fuentes teóricas de persistencia y distingue entre persistencia heredada e intrínseca. La persistencia heredada proviene de otra variable que afecta a la inflación, por ejemplo la brecha del producto. Por otro lado, en la persistencia intrínseca, la inercia inflacionaria guarda una relación estrecha con la persistencia de la variable $\pi_{t-1}$, esto significa que la inflación depende de sus rezagos.

De acuerdo a estos conceptos y a la evidencia empírica la persistencia no es una característica inherente a la inflación, sino más bien un rasgo de este proceso (persistencia intrínseca), no desvinculado al régimen monetario (Levin \& Piger, 2004).

Este trabajo se enfoca en la forma reducida de la persistencia de la inflación ya que se refiere a una propiedad empírica de la medida de inflación observada. No existe una única medida de persistencia de forma reducida. Diferentes trabajos han demostrado una variedad de medida para captar la idea de que la inflación responde poco a poco a los shocks o permanece cerca de su historia reciente.

Siguiendo el trabajo de Fuhrer (2009) se mencionan brevemente las distintas medidas de persistencia de la inflación en forma reducida:

\section{Test de raíces unitaria.}

Con el objetivo de evaluar las características temporales de la inflación y comenzar a evaluar la presencia de quiebres en su valor medio es conveniente utilizar los estadísticos de Dickey-Fuller que evalúan la presencia de una raíz unitaria controlando por la significatividad de la constante y la tendencia, es decir, de los componentes determinísticos.

\section{Tests de Bai-Perron}

Los distintos regímenes monetarios que tuvo la Argentina en el periodo analizado imposibilitan estudiar la serie de inflación sin evaluar la presencia de quiebres en su valor medio, para ello incorporamos los tests de Bai \& Perron (2003), los cuales nos indican la posibilidad de múltiples quiebres estructurales en el proceso dinámico de la inflación. Esta metodología propone tests para evaluar la hipótesis nula de no quiebres frente a la hipótesis alternativa de presencia de múltiples quiebres. También estos tests permiten tener en cuenta quiebres tanto en media como en componente autorregresivo.

\section{Suma de los coeficientes autoregresivos de la inflación}

El modelo que utilizamos para calcular la persistencia de la inflación es el propuesto por Andrews \& Chen (1994), quienes enfatizan que una medida ampliamente aceptada de persistencia es la suma de los coeficientes autorregresivos de un proceso $\operatorname{AR}(p)$. 


$$
\begin{array}{r}
\pi_{t}=\alpha+\sum_{i=1}^{p} \beta_{i} \pi_{t-i}+\eta_{t} \\
\rho=\sum_{i=1}^{p} \beta_{i}
\end{array}
$$

Donde $\pi_{t}$ es la inflación; $\eta_{t}$ representa al error; y $\rho$ es la suma de los coeficientes autorregresivos, es decir, lo que Andrews \& Chen proponen como medida de persistencia de la inflación.

De acuerdo a la definición de persistencia y como describe Marques (2004), una representación adecuada de ese proceso es reescribir la ecuación (1) en término de desvíos de su valor de largo plazo:

$$
\begin{gathered}
\pi_{t}-\mu=\sum_{i=1} \beta\left(\pi_{t-i}-\mu\right)+\eta_{t} \\
\mu=\frac{\alpha}{1-\rho}
\end{gathered}
$$

Donde la ecuación (4) representa la media no condicional de la inflación.

La ecuación (3) también puede ser reescrita como un modelo de corrección al equilibrio reparametrizada del siguiente modo:

$$
\left(\pi_{t}-\mu\right)=\sum_{i=1}^{p-1} \varphi_{i} \Delta\left(\pi_{t-i}-\mu\right)+\rho\left(\pi_{t-1}-\mu\right)+\eta_{t}
$$

Donde $\mu$ es la media no condicional de la inflación, $\varphi_{i}=-\sum_{i=1+j}^{p} \beta_{i}$, y $\rho$ es la suma de los coeficientes autoregresivos el cual puede obtenerse directamente si estimados el modelo para la serie de desviaciones de la media $\left(\pi_{t}-\mu\right)$. Para este análisis debemos suponer que que $\pi_{t}$ es una series estacionaria con $0<\rho<1$, también es necesario aclarar que cuanto mas elevado es el valor absoluto de $\rho$, mas lentamente la inflación retorna a su valor de equilibrio.

\subsection{Dinámica inflacionaria. Enfoque Multivariado: VAR.}

Se estudia la dinámica de corto plazo de las variables seleccionadas estimando sistemas de Vectores Autorregresivos (VAR), siguiendo la metodología propuesta por Sims (1980). Estos modelos, en los que todas las variables pueden ser tratadas como endógenas, suelen ser utilizados para describir las relaciones entre los agregados macroeconómicos en el ciclo.

La especificación general de un modelo VAR está dada por la siguiente expresión: 


$$
Y_{t}=c+\emptyset_{1} Y_{t-1}+\emptyset_{2} Y_{t-2}+\cdots+\emptyset_{p} Y_{t-p}+\varepsilon_{t}
$$

Donde $Y_{t}$ es un vector de variables objeto de predicción, $Y_{t-1}, Y_{t-2}, \ldots, Y_{t-p}$ son los vectores de rezagos de las variables del sistema, los coeficientes $\emptyset$ son matrices de coeficientes a estimar, y $\varepsilon$ es un vector de perturbaciones aleatorias que cumplen los siguientes supuestos:

$$
\begin{aligned}
E\left(\varepsilon_{t}\right) & =0 \quad(7) \\
E\left(\varepsilon_{t} \varepsilon_{\tau}\right) & =\left\{\begin{aligned}
\Omega_{N x N}, & \text { para } t=\tau \\
0, & \text { para } t \neq \tau
\end{aligned}\right.
\end{aligned}
$$

Esta especificación puede ser estimada de manera consistente por mínimo cuadrados ordinarios (MCO) teniendo en cuenta algunas consideraciones:

- Todas las variables del modelo son tratadas simétricamente, siendo explicadas por el pasado de todas ellas, el modelo tiene tantas ecuaciones como variables, y los valores rezagados de todas las variables aparecen como variables explicativas en todas las ecuaciones. Esto significa que cada variable se regresa en función de una constante y $\mathrm{p}$ rezagos de la propia variable así como en $\mathrm{p}$ rezagos de cada una de las otras variables del VAR. Se debe notar que cada regresión tiene las mismas variables explicativas.

- Se puede estimar con bastante precisión los elementos globales del modelo, o el efecto global de una variable sobre otra, lo que se resume en tests de anticipación temporal.

- EI VAR no es una forma estructural, no se pueden hacer interpretaciones de coeficiente individuales de los distintos rezagos, ni llevar a cabo contrastes de hipótesis sobre coeficientes individuales, solo se busca una adecuada representación de los datos.

- La metodología VAR brinda información sobre la dinámica de corto plazo presente en los datos a través del cálculo de (i) funciones impulso-repuesta, que permiten cuantificar la respuesta de las variables endógenas a un shock (impulso) sobre alguna de ella y (ii) descomposiciones de varianza, que indican qué proporción de la varianza de cada variable del sistema es explicada por shock sobre el resto de las variables y que proporción por ella misma

\section{La dinámica inflacionaria en Argentina}

Como se ha remarcado el supuesto de un valor de equilibrio constante para la tasa de inflación no es razonable en el caso Argentino. Al respecto, Capistrán \& Ramos-Francia (2006), proveen evidencia sobre persistencia de la inflación para los diez países más grandes de América Latina y encuentran que en el caso argentino el grado de persistencia 
de la inflación se habría reducido entre enero de 1980 y febrero de 2007. También para el caso de Argentina D'Amato, Garegnani, \& Sotes (2008) y D'Amato \& Garegnani (2013) encuentran quiebres en la tasa media de inflación que resultan coincidentes con cambios de régimen: el ingreso a un régimen de alta dominancia fiscal luego del episodio inflacionario de 1975, dominancia que se acentúa luego de la crisis de la deuda en 1982, hasta derivar en dos episodios hiperinflacionarios en 1989 y 1990, la adopción de la Convertibilidad en 1991 y el abandono de ese régimen en enero de 2002. El gráfico (1) muestra una representación de la tasa de inflación para el periodo 1980-2013 que es el periodo estudiado en el presente trabajo.

Tasa de Inflación. 1980-2013

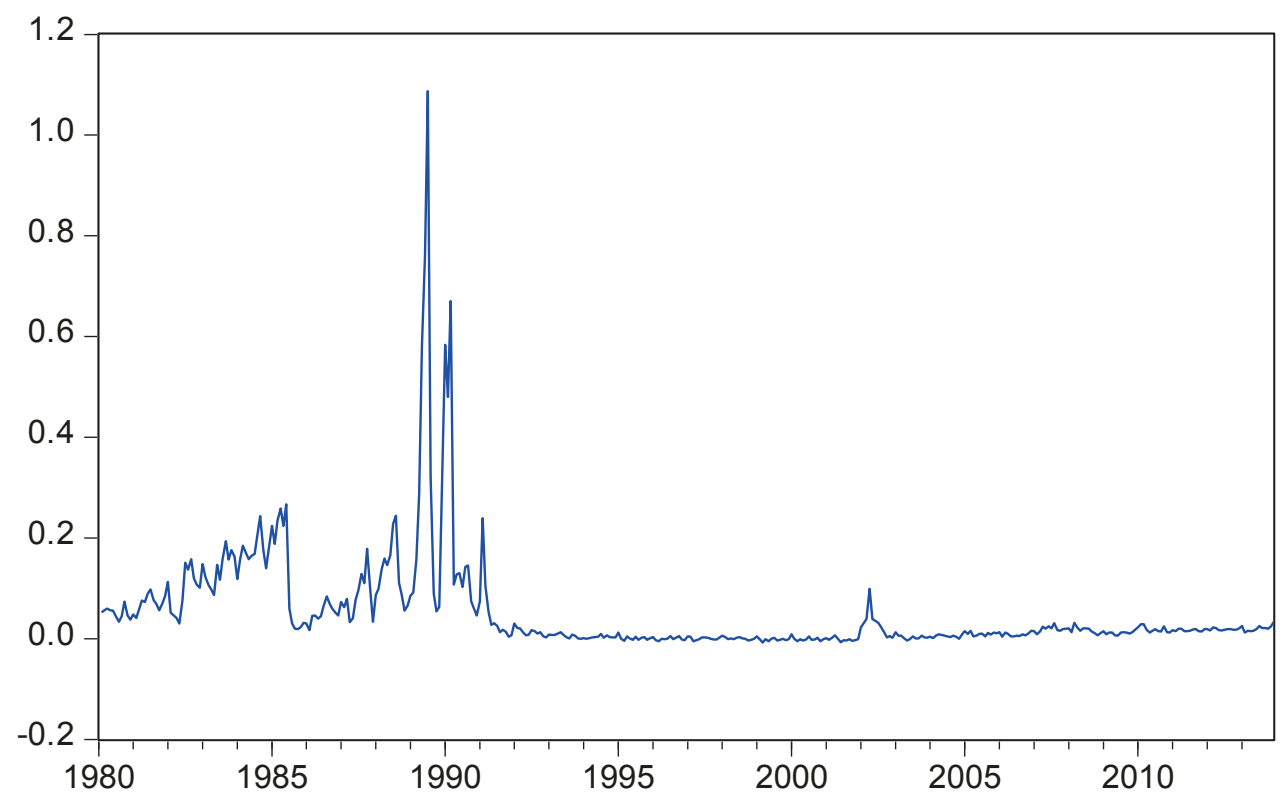

Como se puede observar en el gráfico, la Argentina pasó por periodos de alta inflación, como se observa en los años 80 , una hiperinflación a fines de esta década y periodos de baja inflación desde los años 90 en adelante, con un quiebre en el año 2002.

A comienzo de los años 80 el país incrementaba su deuda debido a las atractivas tasas de interés internacionales, paso de 5 mil a 44 mil millones de dólares entre 1973 y 1982. Los países desarrollados entraron en recesión, lo que provoco una baja en sus compras y atacaron sus desequilibrios estructurales a través de una estricta política monetaria elevando las tasas a más de doble. También se produjo una fuga de capitales del orden de los 20 mil millones de dólares, que se reflejaron en la caída de las reservas del Banco Central. Esto trajo consecuencias en el país, el peso fue devaluado y el gobierno se hizo cargo de una parte importante de la deuda externa privada lo que incremento los desequilibrios fiscales preexistentes. 
A comienzo de 1985 se toman nuevas medidas relacionadas con ajustes de tarifas y del tipo de cambio. Entre ellas, se reduce el $12 \%$ el gasto público, aumento en las tarifas de los servicios públicos y los combustibles, la suspensión de las obras públicas y el propósito de incorporar capital privado en diversas empresas estatales. Poco después el gobierno implementa el Plan Austral el cual intenta estabilizar la inflación inercial. Los efectos iniciales del plan fueron favorables, mejoraron la producción, el empleo y los salarios reales.

Este plan no se pudo sostener en el tiempo, los precios siguieron creciendo y a fines de los 80 Argentina entra en un periodo hiperinflacionario. Los precios subieron un $1700 \%$, el tipo de cambio de devaluó casi cuatro veces y los salarios reales cayeron el $30 \%$.

A principios de 1991 el gobierno encontró condiciones externas distintas a las de la década anterior. Los intereses impagos de la deuda con la banca privada fueron reprogramados a 15 años y los de capital a 30 años. La baja tasa de interés en los mercados internacionales reiniciaron la corriente de capitales de corto plazo hacia el país y al mismo tiempo las privatizaciones de empresas públicas atraían la inversión privada directa.

En este contexto externo y sobre la base del aumento de las reservas del Banco Central se realizó una reforma monetaria fundada en un sistema de caja de conversión con un tipo de cambio fijo, de un peso por un dólar. Gracias a esto lograron anclar las expectativas inflacionarias y producir una reducción permanente de la tasa de inflación, que fue en promedio para esa década, cercana a cero.

En el 2001 se produce una crisis financiera y externa que derivo en la caída del régimen de convertibilidad y la devaluación del peso de un 300\% que llevo a un cambio de precios relativos y esto dio lugar a una aceleración de la inflación, que alcanzo un pico en abril de 2002 y luego se redujo. A partir del 2007 la inflación comienza nuevamente a incrementarse debido a un aumento en el gasto público y a políticas que buscan expandir la demanda agregada. Esto trae como consecuencia un incremento en la emisión monetaria y en el tipo de cambio nominal.

\section{Resultados}

En esta sección se comentan los resultados del análisis desarrollado tanto en el modelo univariado de persistencia de la inflación como en el multivariado VAR. La breve descripción del comportamiento histórico de la inflación en Argentina en la sección precedente, sugeriría la presencia de importantes quiebres estructurales y episodios atípicos como puede ser las hiperinflaciones. A pesar de estas características de la dinámica inflacionaria es razonable esperar que los shocks sobre esa variable no tengan un efecto permanente, en la medida 
que la política económica en general y la monetaria en particular, actúen brindándole a la economía algún ancla nominal que logre estabilizar la inflación.

Marques (2004) y Levin \& Piger (2004) indican que si los quiebres en la tasa media de inflación no se toman en cuenta, es posible que el nivel estimado de persistencia sea espúreamente alto. Coincidentemente, esos quiebres aparecen asociados a cambios en el régimen monetario (Altissimo et al. (2006)). Es justamente por este motivo que construimos variables dummies para cada periodo de régimen monetario a los fines de estudiar adecuadamente la dinámica inflacionaria sujeta al contexto económico, monetario y político subyacente.

\subsection{Enfoque Univariado: Persistencia de la Inflación}

Estudiamos en esta sección las propiedades de serie de tiempo de la inflación, expresada en valores mensuales, y evaluamos la presencia de quiebres en su valor medio mediante el análisis descriptivo.

\subsubsection{Periodo 1980-2013}

\subsubsection{Análisis descriptivo}

La tabla (1) muestra la media y el desvío estándar de la inflación en los periodos seleccionados. En el apéndice 2 se describe brevemente cada periodo.

Tabla (1). Media y desvío estándar de la tasa de inflación

\begin{tabular}{|c|c|c|}
\hline Periodo & Media & $\begin{array}{c}\text { Desvío } \\
\text { estándar }\end{array}$ \\
\hline 1980:1-1989:3 & 0,1034 & 0,0619 \\
\hline 1989:4-1990:3 & 0,4430 & 0,3132 \\
\hline 1990:4-1991:2 & 0,1136 & 0,0537 \\
\hline 1991:3-1992:12 & 0,0208 & 0,0221 \\
\hline 1993:1-2007:2 & 0,0046 & 0,0106 \\
\hline 2002:1-2002:9 & 0,0371 & 0,0246 \\
\hline 2002:10-2007:2 & 0,0065 & 0,0045 \\
\hline $1980: 1-2007: 2$ & 0,0590 & 0,1126 \\
\hline $2007: 1-2013: 12$ & 0,0175 & 0,0054 \\
\hline $1980: 1-2013: 12$ & 0,0507 & 0,1020 \\
\hline
\end{tabular}

Se puede observar de la Tabla 1 que tanto la media como la volatilidad de la inflación fueron cambiantes a lo largo de la muestra y que en ese sentido los estadísticos descriptivos para el período completo son poco informativos acerca del comportamiento de la inflación entre 1980 y 2013. 
En el periodo 1980:1-1989:3 la tasa promedio de inflación fue del 10\% la cual desemboca a fin de la década en una tasa cercana al $45 \%$ con una volatilidad de $31 \%$. Luego en los años 90 hay un periodo de desinflación al comienzo del periodo el cual lleva a una época de baja inflación del 0,5\% mensual en la en el periodo 1993-2007. En este periodo se encuentra la devaluación del año 2002 con una inflación promedio del 3,7\%.

A partir del 2007, la tasa de inflación promedio estuvo en 1,75\%. Mucho de los trabajos que usan la variable de inflación para su análisis finalizan en 2007 , este trabajo amplía la muestra desde entonces hasta el año 2013.

\subsubsection{Test de raíces unitarias}

En el apartado 2.1 se menciona este test como una medida para evaluar la existencia de una raíz unitaria en la variable inflación y también como importante para medir la persistencia. Además se utiliza para estudiar las características temporales de la inflación y evaluar la presencia de quiebres en su valor medio. Esta estimación se hace controlando por la significatividad de los componentes determinísticos: la media y la tendencia determinística.

Tabla (2). Estadístico F de Dickey Fuller para raíces unitarias.

\begin{tabular}{|c|c|c|c|c|}
\hline & 1980:1-1989:3 & 1993:1-2007:2 & 2007:1-2013:12 & 1980:1-2013:12 \\
\hline Constante & Significativo** & No significativo & Significativo $^{* * *}$ & Significativo** \\
\hline Tendencia & No significativo & No significativo & No significativo & Significativo** \\
\hline $\mathrm{HO}=$ unit-root & Rechaza * & Rechaza *** & Rechaza $^{* * *}$ & Rechaza ** \\
\hline
\end{tabular}

Se observa en la tabla (2) que en todos los periodos estudiados se rechaza la presencia de raíz unitaria. Con respecto a una media constante igual a 0 , no se rechaza la presencia en el periodo 1993:1-2007:2. Para los demás periodos se rechaza la hipótesis nula de media igual a 0 . En el único periodo que se identifica una tendencia estadísticamente significativa es en el periodo total, 1980:1-2013:12. Con estos resultados señalamos que si bien la inflación no tiene una raíz unitaria, tampoco puede ser considerada como un proceso estacionario, en la medida que se identifican cambios significativos en su valor medio.

\subsubsection{Presencia de quiebres. Análisis recursivo.}

En este apartado se busca identificar la presencia de quiebres en la media y el componente autorregresivo de la inflación a través del análisis recursivo y del test de Bai Perron. Además se observa la relación de los quiebres con la ocurrencia de cambios en regímenes monetarios. 
Estimamos recursivamente la ecuación (1) con un rezago de la inflación y se observa la presencia de quiebres tanto en la constante $(C(1))$ como en el coeficiente autoregresivo (C(2)) utilizando tests de cambio estructural. El gráfico (2) muestra que ambos coeficientes, constante y término autoregresivo se ubican fuera del intervalo de +/- 2 veces los desvíos estándares previos en el entorno de la hiperinflación.

Gráfico (2). Análisis recursivo
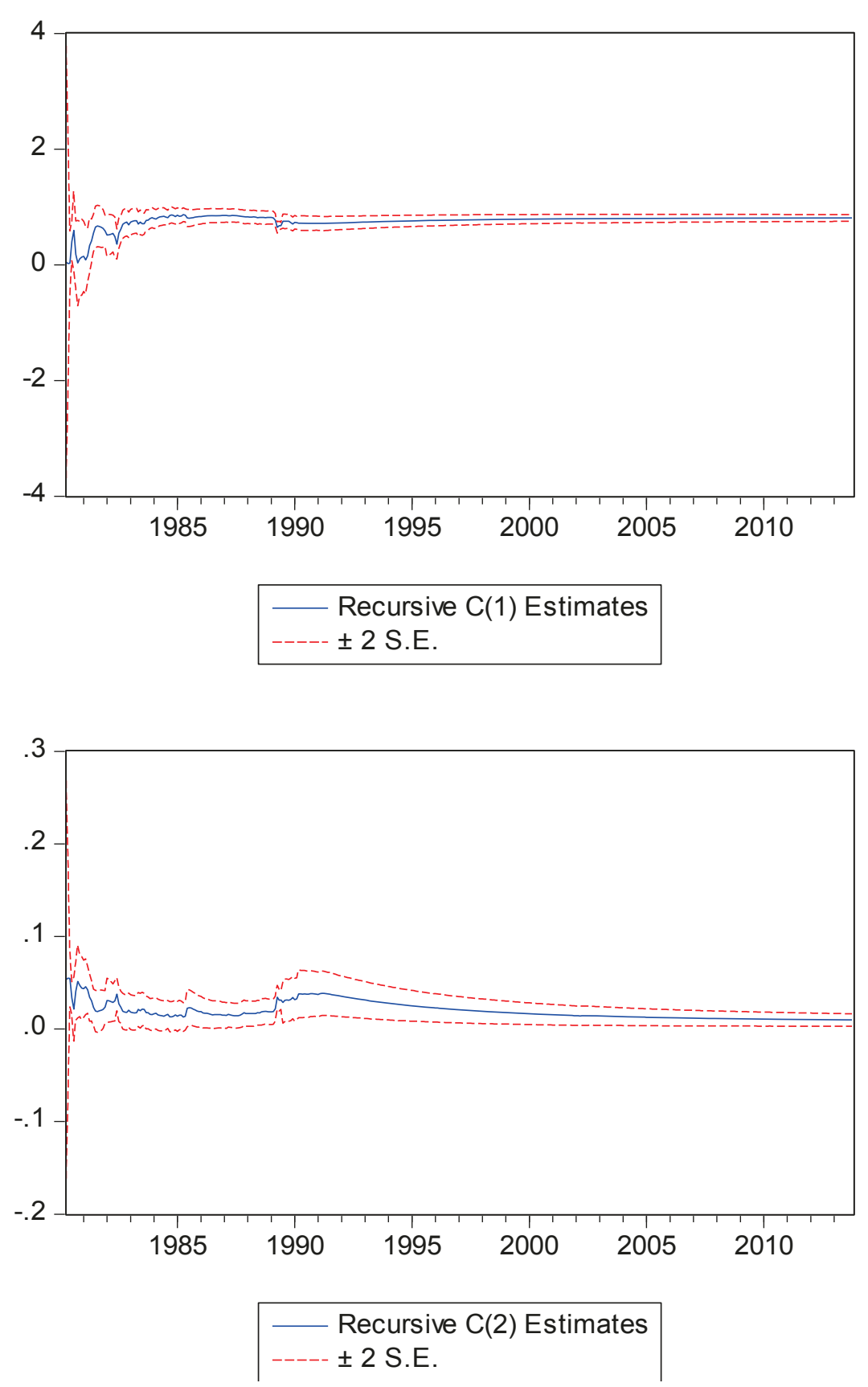

De acuerdo a este análisis, se encuentra cambios en la dinámica de la inflación en el episodio hiperinflacionario, y cerca de la adopción de un régimen cambiario de convertibilidad. Luego de estos episodios no es posible encontrar quiebres en la dinámica de la inflación. 
También el análisis recursivo indica que la no estacionariedad de la inflación mencionada anteriormente, proviene de una media no constante. De acuerdo a esto, se sugiere diferenciar la serie respecto de esa media no constante, que evoluciona a saltos.

\subsubsection{Test Bai y Perron}

Estos tests desarrollados por Bai \& Perron (2003) nos permiten estudiar la posibilidad de la existencia de múltiples quiebres estructurales en el proceso dinámico de la inflación. Esta metodología propone tests para evaluar la hipótesis nula de no quiebres contra la alternativa de la presencia de múltiples quiebres.

El test que se lleva a cabo es aquel más general que permite tanto cambios en media como en el componente autorregresivo.

Tabla (3). Test Bai y Perron 1980-2013

\begin{tabular}{|l|r|r|r|}
\hline \multicolumn{4}{|c|}{ Cambios en media $\left(\boldsymbol{\alpha}_{1}\right)$ y autorregresivo $\left(\boldsymbol{\beta}_{1}\right)$} \\
\hline Test \\
\hline Break Test & F-statistic & Scales F-statistic & Critical Value \\
\hline 0 vs $1^{\text {* }}$ & 98,47 & 196,94 & 11,47 \\
\hline 1 vs 2 & 5,5 & 11,02 & 12,95 \\
\hline Nro de quiebres \\
\hline 1 \\
\hline \multicolumn{4}{|l|}{ Fechas de quiebres } \\
\hline Agosto de 1989 \\
* Significativo al 5\%
\end{tabular}

El test identifica un solo quiebre en agosto de 1989, el cual está en el entorno del primer episodio hiperinflacionario. La dramática volatilidad implicada por el episodio hiperinflacionario limita la posibilidad de identificar quiebres adicionales en la serie de inflación, es por ello que es tan interesante complementar estos tests con la metodología de Marques (2004) que se desarrolla en la sección siguiente.

\subsubsection{Persistencia inflacionaria.}

Siguiendo a Marques (2004) construimos una media no constante para la tasa de inflación de acuerdo a los modelos del apartado 2.1. En este análisis utilizamos variables dummies que identifican cambios en la tasa media de inflación de acuerdo a lo sugerido por el análisis descriptivo ${ }^{2}$. En este modelo también se evalúa la presencia de una tendencia determinística positiva en el periodo de alta inflación.

\footnotetext{
${ }^{2}$ Donde d1 es una variable dummy para el periodo 1980:1-1989:3, d2 para 1989:7-1990:3, d3 para 1990:4-1991:2, d4 para 1991:3-2001:12, d5 para 2002:1-2002:8, d6 para 2002:9-2006:12, d7 para 2007:1-2013:12 y t1 es una tendencia determinística para 1980:1-1989:3.
} 
Ecuación (9)

\begin{tabular}{|c|c|c|}
\hline \multicolumn{3}{|c|}{ Variable dependiente: $\pi_{t}$} \\
\hline Variable & Coeficiente & $\begin{array}{c}\text { Error } \\
\text { Std. }\end{array}$ \\
\hline $\mathrm{c}$ & 0,5434 & 0,0354 \\
\hline $\mathrm{d} 1$ & $-0,4586$ & 0,0374 \\
\hline $\mathrm{d} 2$ & $-0,1338$ & 0,0409 \\
\hline $\mathrm{d} 3$ & $-0,4298$ & 0,0400 \\
\hline $\mathrm{d} 4$ & $-0,5392$ & 0,0359 \\
\hline $\mathrm{d} 5$ & $-0,5033$ & 0,0416 \\
\hline $\mathrm{d} 6$ & $-0,5370$ & 0,0365 \\
\hline $\mathrm{d} 7$ & $-0,5259$ & 0,0361 \\
\hline $\mathrm{t} 1$ & 0,00034 & 0,0002 \\
\hline
\end{tabular}

Si observamos los resultados de la ecuación (9), el valor constante de $54,34 \%$ es la media de la tasa de inflación del periodo de hiperinflación (1989:4 - 1989:6). Otro periodo relevante de alta inflación es el comprendido entre 1989:7 y 1990:3 el cual arroja una tasa media de inflación del $41 \%$. Luego de la adopción del esquema monetario de la Convertibilidad, la tasa de inflación tuvo una reducción importante, resultando estadísticamente no distinta de cero $(0,4 \%)$.

Luego del abandono de la convertibilidad en enero del 2002, se produjo una aceleración inflacionaria luego de la abrupta devaluación del peso, llegando a picos del 10\% para luego desacelerarse, en promedio la inflación en el periodo 2002:9-2006:12 fue del 0,6\%.

Para el periodo de 2007:1-2013-12 la tasa media de inflación fue del 1,8\%, este incremento podría estar asociado a las políticas económicas actuales de expansiones del gasto público y de la demanda agregada.

Adicionalmente se estudió la presencia de una tendencia determinística positiva en el periodo de alta inflación, década del 80 la cual se observa en el gráfico (3) la cual ha resultado ser estadísticamente significativa al $10 \%$. 
Gráfico (3).

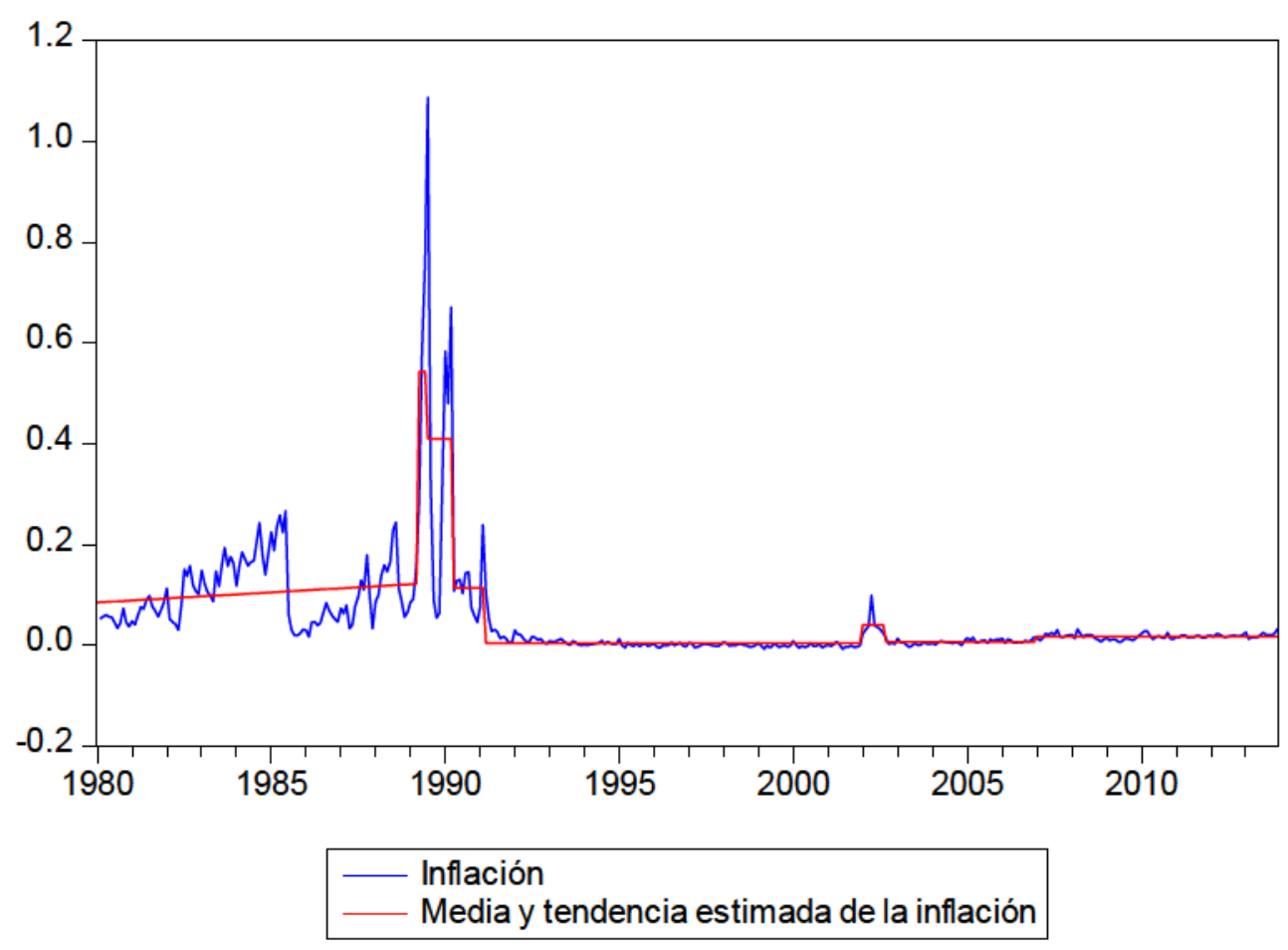

En la tabla (3) mostramos las medidas de persistencia obtenidas de la estimación de la ecuación (1) utilizando una media constante y una no constante.

Tabla (3).

\begin{tabular}{|c|c|c|}
\hline & Media constante & Media no constante \\
\hline$\rho$ & 0,81 & 0,51 \\
\hline Error est. & 0,030 & 0,038 \\
\hline & $(1$ rezago) & (1 rezago) \\
\hline
\end{tabular}

La tabla (3) indica que la inflación es un proceso altamente persistente teniendo en cuenta una media constante, 0,81 . En cambio si suponemos que la media es no constante, la persistencia baja a 0,51 . De acuerdo a estos resultados afirmamos que existen múltiples quiebres en la media de inflación y que si se controla por ellos la inflación aparece como un proceso bastante menos persistente que si se considera una media constante.

Otra cuestión a investigar en la dinámica de la inflación, asociada a los cambios en la media, es si se identifican cambios en el componente autorregresivo de la serie. Tanto el análisis recursivo como el test de Bai-Perron han dado cuenta de la presencia de cambios en los coeficientes autorregresivos de la inflación asociados a quiebres en la tasa media de inflación. 
Para finalizar entonces el análisis de persistencia, y una vez que obtuvimos la media no constante de la tasa de inflación, calculamos los desvíos de la inflación respecto de esa media a la que llamaremos $z_{t}$ para luego estimar la ecuación (5) y calcular así una medida de persistencia que considera una media no constante. Para ello se estima un modelo autorregresivo de $z_{t}$ incluyendo dummies que multiplican a los niveles y diferencias de $z_{t}$. $A$ continuación se muestra el modelo (ecuación 10) y la tabla (4) presenta las medidas de persistencias obtenidas de la estimación de dicha ecuación.

Ecuación (10)

\begin{tabular}{|c|c|c|}
\hline \multicolumn{3}{|c|}{ Variable dependiente: $\mathrm{z}_{\mathrm{t}}$} \\
\hline Variable & Coeficiente & Error Std. \\
\hline $\mathrm{z}_{\mathrm{t}-1} \mathrm{~d} 1$ & 0,5191 & 0,0409 \\
\hline $\mathrm{z}_{\mathrm{t}-1} \mathrm{~d} 3$ & 0,3935 & 0,0535 \\
\hline $\mathrm{z}_{\mathrm{t}-1} \mathrm{~d}$ & $-0,6962$ & 0,0804 \\
\hline$\Delta \mathrm{z}_{\mathrm{t}-1}$ & 0,3518 & 0,0248 \\
\hline$\Delta \mathrm{z}_{\mathrm{t}-1} \mathrm{~d} 1$ & $-0,3076$ & 0,0588 \\
\hline$\Delta \mathrm{z}_{\mathrm{t}-1} \mathrm{~d} 4^{\prime}$ & $-0,1755$ & 0,1134 \\
\hline$\Delta \mathrm{z}_{\mathrm{t}-1} \mathrm{~d} 4 "$ & $-0,5047$ & 0,1878 \\
\hline$\Delta \mathrm{z}_{\mathrm{t}-1} \mathrm{~d} 7$ & $-0,3666$ & 0,3992 \\
\hline djul85 & $-0,1947$ & 0,0205 \\
\hline variables dummies periodo & & \\
\hline hiperinflación & & \\
\hline
\end{tabular}

Tabla (4)

\begin{tabular}{|c|c|}
\hline Sub-periodo & $\boldsymbol{\rho}$ \\
\hline 1980:1-1989:3 & 0,957 \\
\hline 1989:4-1990:3 & 0,871 \\
\hline 1990:4-1991:2 & 0,175 \\
\hline 1991:3-1992:12 & 0,695 \\
\hline 1993:1-2006:12 & 0,366 \\
\hline $2007: 1-2013: 12$ & 0,504 \\
\hline
\end{tabular}

De acuerdo al modelo estimado ${ }^{3}$ se encuentra que la inflación fue un proceso altamente persistente $(0,96)$ en el periodo de inflación elevada, 1980:1-1989:3 y fue de 0,87 en el periodo 1989:4-1990:3; más alta que cuando se suponía una media constante para toda la muestra. Los períodos subsecuentes que abarcan la transición (1990:4 - 1991:2) y el que siguió a la implementación del régimen de Convertibilidad (1991:3-1992:12) si bien no son interesantes en sí mismos para medir el grado de persistencia, es necesario controlar por ellos para medir adecuadamente la persistencia en los periodos relevantes al estudio.

\footnotetext{
${ }^{3}$ Donde d4' es una variable dummy para el periodo 1991:3-1992:12, d4" para 1993:1-2006:12
} 
También observamos que en periodo denominado de inflación baja, periodo de convertibilidad y posterior devaluación, la persistencia se redujo $(0,37)$. El último periodo, 2007:1-2013:12, que será estudiado con más detalle a partir del apartado siguiente, presenta una medida de persistencia superior a la observada desde el año 1993 que es de 0.50 .

\subsubsection{Periodo 2003-2013}

Como se observó en los resultados previos, la fuerte volatilidad implicada por el episodio hiperinflacionario parecería limitar la posibilidad de identificar quiebres en la serie de inflación luego del periodo de convertibilidad y devaluación de la moneda (adopción de un régimen de flotación administrada en 2002) luego de la crisis de 2001. Si bien se ha identificado un quiebre en el año 2007 por su significatividad en las regresiones de cambio en media y en coeficientes autorregresivos, este quiebre no se observaba en el análisis recursivo ni en el test de Bai-Perron del análisis previo. Es así que queremos confirmar que existe dicho quiebre en el contexto del período 2003-2013 período con menor volatilidad de la inflación.

En el Gráfico (4), se muestra la tasa de inflación para el subperíodo en estudio advirtiendo un aumento en su valor medio a partir del año 2007 con la sola inspección visual.

Tasa de Inflación. 2003-2013

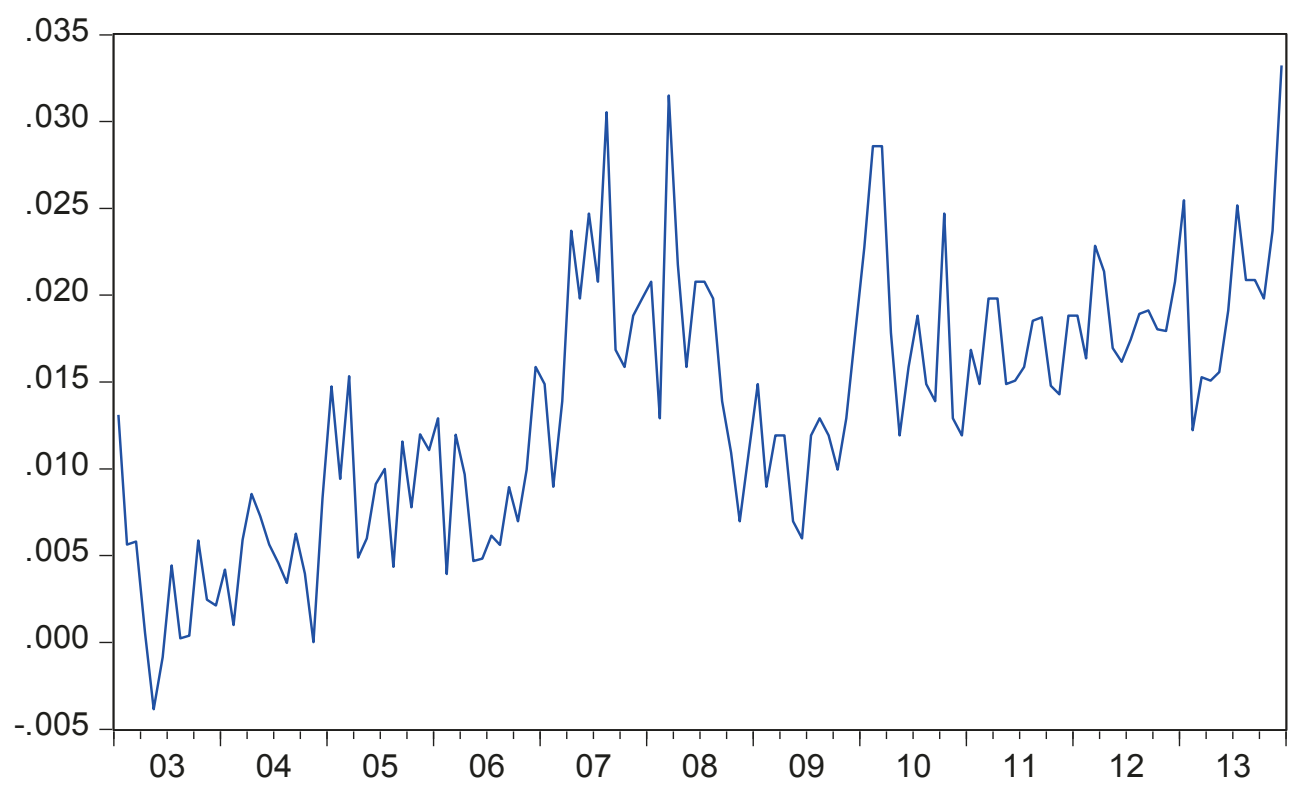

\subsubsection{Análisis descriptivo}

El análisis de este subperíodo permite estudiar en qué medida la adopción del régimen de flotación administrada en enero de 2002 implicó cambios en la dinámica de la inflación no capturados al considerar el período completo de estudio. La tabla (5) muestra la media y el 
desvío estándar de la inflación en dicho período, la subdivisión en el año 2007 está motivada por la observación del Gráfico (4).

Tabla (5). Media y desvío estándar de la tasa de inflación

\begin{tabular}{|c|c|l|}
\hline Periodo & Media & $\begin{array}{c}\text { Desvío } \\
\text { estándar }\end{array}$ \\
\hline 2003:1-2006:12 & 0.0065 & 0.0044 \\
\hline 2007:1-2013:12 & 0.0175 & 0.0054 \\
\hline 2003:1-2013:12 & 0.0135 & 0.0073 \\
\hline
\end{tabular}

En el periodo completo, 2003:1-2013:12, la tasa media de inflación fue del 1,35\%, aumentando al 1,75\% en el periodo 2007:1-2013:12. En el primer periodo, la tasa media de inflación no supera un digito, es del 0,6\%.

\subsubsection{Test de raíces unitarias.}

Debido a la brevedad del período realizamos el test de raíz unitaria para el período completo, encontrando que se rechaza la hipótesis nula en favor de la alternativa, rechazándose también la hipótesis de media igual a 0 . Se identifica una tendencia estadísticamente significativa en el periodo 2003:1-2013:12.

Tabla (6). Estadistico F de Dickey Fuller para raíces unitarias.

\begin{tabular}{|c|c|}
\hline & 2003:1-2013:12 \\
\hline Constante & Significativo** $^{* *}$ \\
\hline Tendencia & Significativo*** \\
\hline $\mathrm{H} 0=$ unit-root & Rechaza $^{* * *}$ \\
\hline${ }^{* * *} \%$ significatividad \\
${ }^{* *} 5 \%$ significatividad \\
${ }^{*} 10 \%$ significatividad
\end{tabular}

\subsubsection{Presencia de quiebres. Análisis recursivo.}

Estimamos la ecuación (1) con un rezago de la inflación para el periodo 2003-2013 y se observa la presencia de quiebres tanto en la constante como en el coeficiente autoregresivo, utilizando el test de cambio estructural.

El gráfico (5) muestra que ambos coeficientes, constante y término autoregresivo se ubican fuera del intervalo de +/- 2 veces los desvíos estándares previos a partir del año 2007 . 
Gráfico (5). Análisis recursivo
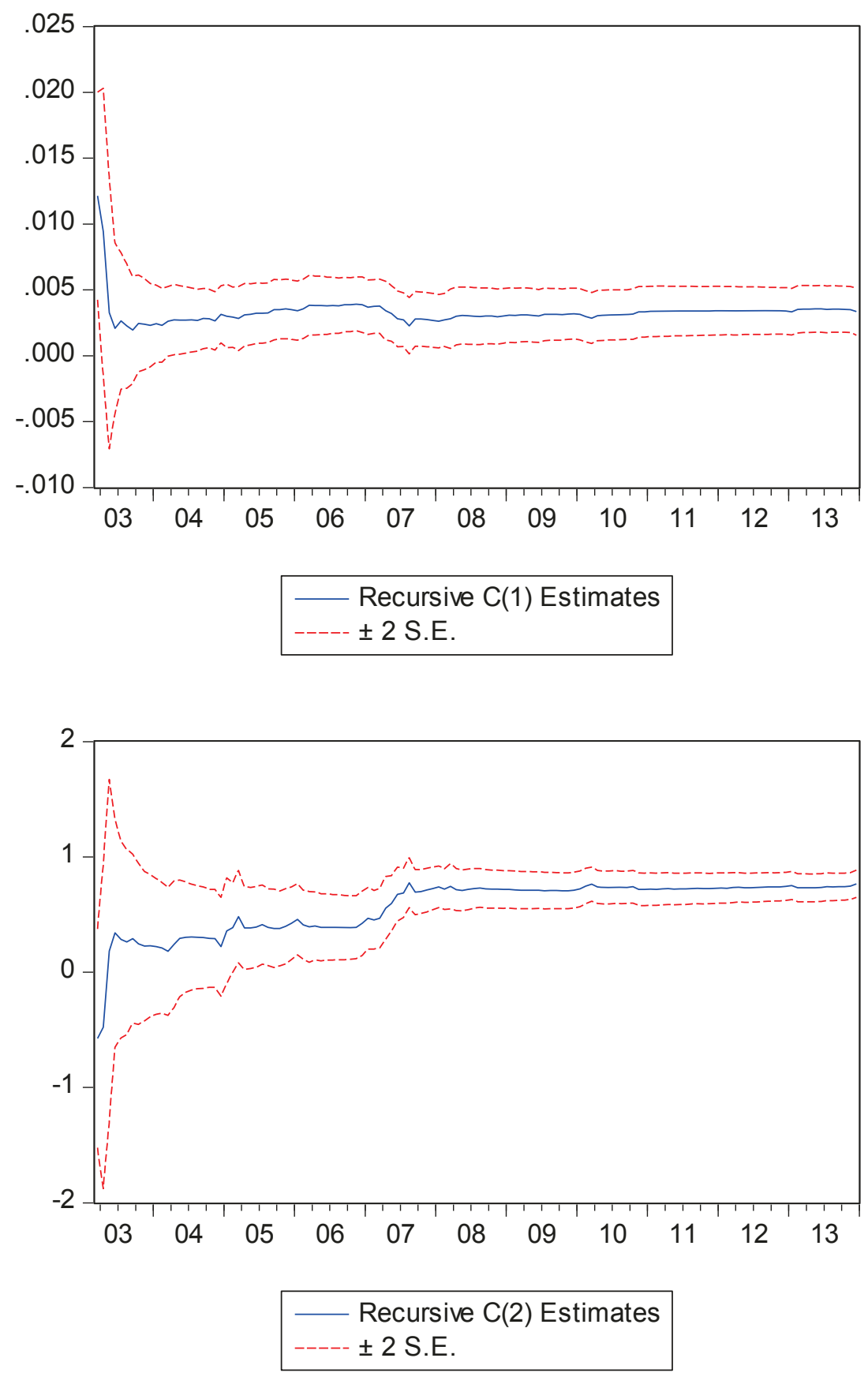

\subsubsection{Test Bai y Perron}

Como en el apartado 4.1.1.4, el test que se lleva a cabo es aquel más general que permite tanto cambios en media como en el componente autorregresivo. La tabla (7) muestra los resultados: 
Tabla (7). Test Bai y Perron 2003-2013

\begin{tabular}{|c|c|c|c|}
\hline \multicolumn{4}{|c|}{ Cambios en media $\left(\alpha_{1}\right)$ y autorregresivo $\left(\beta_{1}\right)$} \\
\hline \multicolumn{4}{|l|}{ Test } \\
\hline $\begin{array}{l}\text { Break } \\
\text { Test }\end{array}$ & \begin{tabular}{|l|}
$\begin{array}{l}F- \\
\text { statistic }\end{array}$ \\
\end{tabular} & $\begin{array}{l}\text { Scales F- } \\
\text { statistic }\end{array}$ & $\begin{array}{l}\text { Critical } \\
\text { Value }\end{array}$ \\
\hline 0 vs $1^{*}$ & 12,34 & 24,68 & 11,47 \\
\hline 1 vs 2 & 4,71 & 9,43 & 12,95 \\
\hline \multicolumn{4}{|c|}{ Nro de quiebres } \\
\hline \multicolumn{4}{|l|}{1} \\
\hline \multicolumn{4}{|c|}{ Fechas de quiebres } \\
\hline Noviemb & de 2006 & & \\
\hline
\end{tabular}

* Significativo al $5 \%$

Los resultados muestran que el quiebre capturado para el período completo se verifica cercano al del análisis recursivo pues en el test de quiebre estructural de Bai-Perron es a partir de noviembre de 2006.

\subsubsection{Persistencia inflacionaria}

Luego de haber identificado la presencia de al menos un quiebre en la tasa media de inflación en el entorno del inicio del año 2007, siguiendo la metodología utilizada para todo el período, construimos una media no constante para la tasa de inflación ${ }^{4}$.

\section{Ecuación (11)}

\begin{tabular}{|c|c|c|}
\hline \multicolumn{3}{|c|}{ Variable dependiente: $\pi_{\boldsymbol{t}}$} \\
\hline Variable & Coeficiente & $\begin{array}{c}\text { Error } \\
\text { Std. }\end{array}$ \\
\hline $\mathrm{C}$ & 0,0052 & 0,0009 \\
\hline $\mathrm{d} 1^{\prime}$ & 0,0073 & 0,0016 \\
\hline $\mathrm{t} 1^{\prime}$ & 0,00006 & 0,00002 \\
\hline
\end{tabular}

Si observamos los resultados de la ecuación (11), el valor constante de $0,52 \%$ es la media mensual de la tasa de inflación del periodo 2003- 2006. Para el periodo 2007-2013, la tasa media de inflación mensual es del $1,3 \%$. Es decir, una vez que consideramos el período 2003-2013 separadamente, es posible identificar un quiebre en media a partir del año 2007. Adicionalmente se estudió la presencia de una tendencia determinística positiva en todo el subperíodo, la cual se observa en el gráfico (6) y resulta estadísticamente significativa.

\footnotetext{
${ }^{4}$ Donde d1' es una variable dummy para el periodo 2007:1-2013:12 y $\mathrm{t} 1$ ' es una tendencia determinística para 2003:1-2013:12.
} 
Gráfico (6).

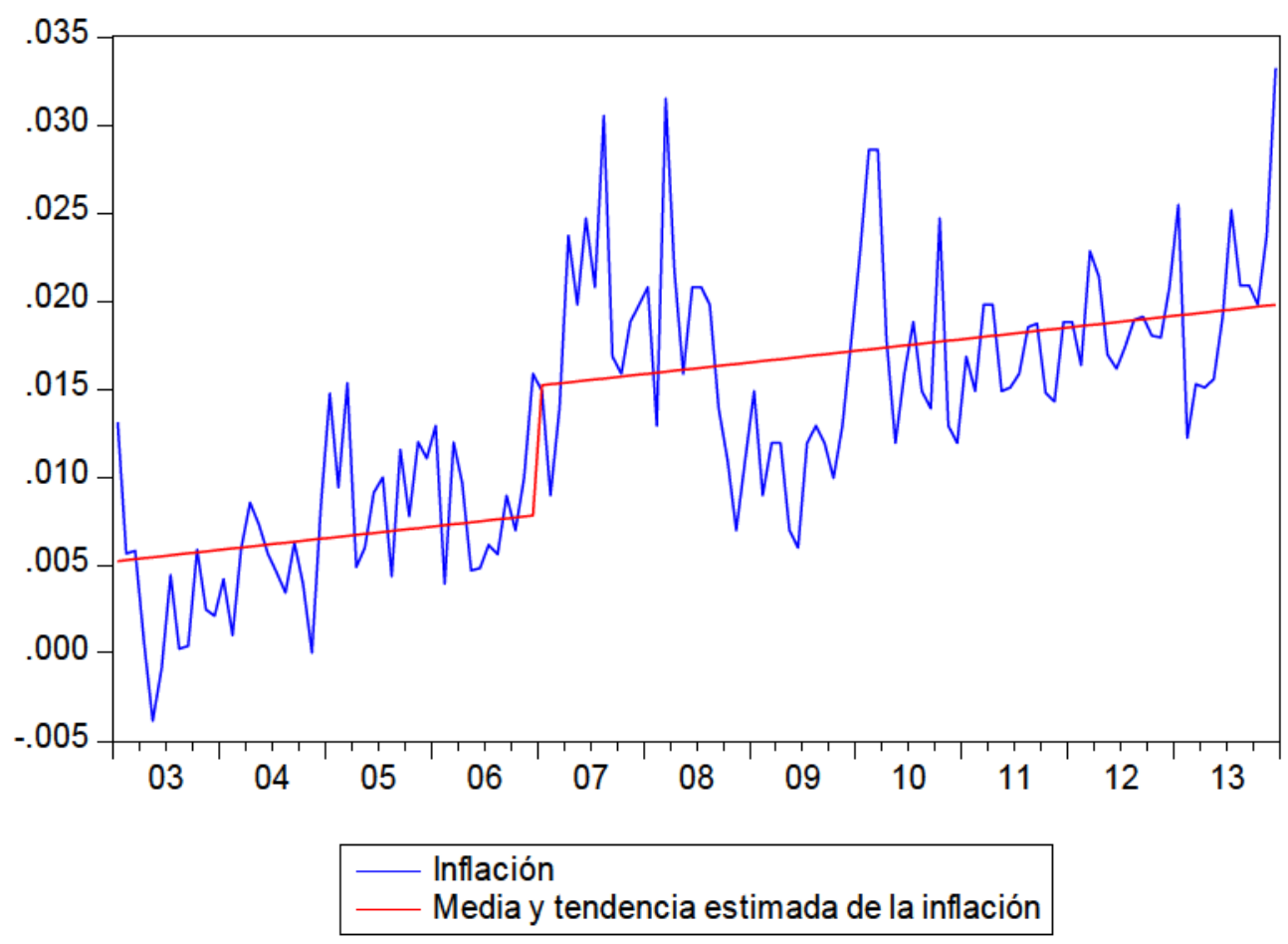

En la tabla (8) mostramos la medida de persistencia obtenidas de la estimación de la ecuación (1) utilizando una media constante y una no constante para el subperíodo 20032013.

Tabla (8)

\begin{tabular}{|c|c|c|}
\hline & Media constante & Media no constante \\
\hline$\rho$ & 0.77 & 0.49 \\
\hline Error est. & 0.059 & 0.082 \\
\hline & $(1$ rezago) & (1 rezago) \\
\hline
\end{tabular}

La tabla (8) indica que la inflación es un proceso altamente persistente teniendo en cuenta una media constante, 0,77 . En cambio si suponemos que la media es no constante, la persistencia baja a 0,49. De acuerdo a estos resultados afirmamos que existe un quiebres en la media de inflación y que si se controla por el la inflación aparece como un proceso bastante menos persistente que si se considera una media constante.

Para finalizar entonces el análisis de persistencia, y repitiendo la metodología del apartado 4.1.1.5, se calculan los desvíos de la inflación respecto de la media a la que llamaremos $z_{t}$ para luego estimar la ecuación (5) y calcular así una medida de persistencia que considera una media no constante. A continuación se muestra el modelo y la tabla (9) de las medias de persistencias obtenidas de la estimación de dicha ecuación. 
Ecuación (12)

\begin{tabular}{|c|c|c|}
\hline \multicolumn{3}{|c|}{ Variable dependiente: $z_{t}$} \\
\hline Variable & Coeficiente & Error Std. \\
\hline $\mathrm{z}_{\mathrm{t}-1}$ & 0,3906 & 0,1717 \\
\hline $\mathrm{z}_{\mathrm{t}-1} \mathrm{~d} 1^{\prime}$ & 0,1023 & 0,2029 \\
\hline$\Delta \mathrm{z}_{\mathrm{t}-1} \mathrm{~d} 1^{\prime}$ & $-0,0115$ & 0,1053 \\
\hline
\end{tabular}

Tabla (9)

\begin{tabular}{|c|c|}
\hline Sub-periodo & $\boldsymbol{\rho}$ \\
\hline $2003: 1-2006: 12$ & 0,391 \\
\hline $2007: 1-2013: 12$ & 0,481 \\
\hline
\end{tabular}

De acuerdo a este modelo utilizado se encuentra que la inflación es más persistente en el periodo 2007:1-2013:12 con un valor de: 0,48; contra una persistencia de 0,39 en el periodo 2003:1-2006:12. Esto indica un cambio en el proceso de inflación tanto en el término de su valor medio como de su componente autorregresivo.

\subsection{Modelo Multivariado VAR}

Si la inflación fuese un proceso fuertemente persistente, los shocks sobre esta variable tardan mucho tiempo en revertirse y producir una desinflación puede ser un proceso altamente costoso en términos de nivel de actividad. Es por esta razón que luego de estudiar la persistencia de la inflación de manera univariada se procede a introducirla en un contexto multivariado a través de modelos de vectores autorregresivos (VAR) propuestos por Sims (1980) para comprender el vínculo de su dinámica con otras variables macromonetarias de la economía.

Como se ha descripto previamente, estos modelos, en los que todas las variables pueden ser tratadas como endógenas, suelen ser utilizados para describir las relaciones entre los agregados macroeconómicos en el ciclo. La metodología VAR brinda información sobre la dinámica de corto plazo presente en los datos a través del cálculo de (i) funciones impulsorepuesta, que permiten cuantificar la respuesta de las variables endógenas a un shock (impulso) sobre alguna de ella y (ii) descomposiciones de varianza, que indican qué proporción de la varianza de cada variable del sistema es explicada por shock sobre el resto de las variables y que proporción por ella misma.

Por una cuestión de interés de los resultados se ha reducido el período muestral a partir del año 2003 luego de la salida del régimen de Convertibilidad y la adopción de la flotación administrada. 


\subsubsection{Periodo 2003-2013}

El objetivo del uso de este sistema en el trabajo es estudiar la dinámica de corto plazo de las variables tasa de inflación, cantidad de dinero (M1), tasa de interés nominal (TNA), tipo de cambio nominal y PBI.

El cálculo de las funciones impulso-respuesta y las descomposiciones de varianza requiere imponer restricciones que permitan la identificación de los shocks. Se utiliza la descomposición de Cholesky que simplemente requiere de imponer algunas restricciones sobre las relaciones contemporáneas las variables. Se elige deliberadamente esta descomposición debido a que el interés del trabajo es describir la dinámica conjunta de las variables más que hacer análisis de política o pronósticos. El orden impuesto para el cálculo de los impulsos-respuesta utilizando la descomposición de Cholesky es: tasa de interés, cambios en la cantidad de dinero, variaciones del tipo de cambio nominal, cambios en el producto y por último la inflación.

En el apéndice 3 se describen la metodología VAR y los ejercicios de simulación: (i) las funciones de impulso-respuesta y (ii) la descomposición de varianza, cuyos resultados se presentan a continuación. Asimismo en el apéndice 4 se encuentra el análisis descriptivo de las variables y sus gráficos.

\subsubsection{Test de raíces unitarias}

Para comenzar el análisis es necesario evaluar las características temporales de las series a través de los tests de Dickey-Fuller que evalúan la presencia de una raíz unitaria controlando por la significatividad de la constante y la tendencia. La tabla (10) presenta los resultados:

Tabla (10). Estadístico F de Dickey Fuller para raíces unitarias

\begin{tabular}{|l|c|c|c|c|c|}
\hline & Inflación & M1 & TNA & TC & PIB \\
\hline Constante & Significativo* $^{*}$ & Significativo** & Significativo** $^{*}$ & No Significativo & Significativo* \\
\hline Tendencia & Significativo** $^{*}$ & Significativo** & Significativo*** & No Significativo & No Significativo \\
\hline H0=unit-root & Rechaza $^{*}$ & No se Rechaza & Rechaza ${ }^{* * *}$ & No se Rechaza & No se Rechaza \\
\hline Diferenciar & No & $\mathrm{Si}$ & No & $\mathrm{Si}$ & $\mathrm{Si}$ \\
\hline
\end{tabular}

*** $1 \%$ significatividad

** $5 \%$ significatividad

*10\%significatividad

Como se observa en la tabla, las series de inflación y TNA no presentan una raíz unitaria siendo variables que presentan una tendencia determinística. En cambio, las restantes variables del sistema, M1, TC y PIB presentan una raíz unitaria. Todas las variables han sido modificadas de manera de transformarlas en estacionarias para incorporarlas en el sistema VAR. 


\subsubsection{Funciones de Impulso-Respuesta y Descomposición de la Varianza}

La selección del modelo VAR que se presentan aquí, se basa en los criterios de evaluación habituales de normalidad, autocorrelación y heterocedasticidad de los residuos, además de evaluarse la presencia de raíces unitarias que pudieran implicar ausencia de convergencia en los sistemas considerados. La elección de la estructura de rezagos se realizó en base a los criterios de Akaike, Schwarz y Hannan-Quinn.

De acuerdo a lo mencionado anteriormente, se incluyen dos variables dummies que controlan periodos de alta inflación: trimestre 2 de 2008 y trimestre 2 de 2012. Además se incluyen dos rezagos de acuerdo a los criterios de elección.

Los resultados de las funciones impulso respuesta se observan en el gráfico (6) y muestran por su parte, que shocks sobre la tasa de crecimiento del M1 tienen un impacto positivo sobre la inflación. Este impacto se hace significativo con un rezago de un trimestre pero luego perdura por tres trimestres. Asimismo la tasa de inflación responde negativamente a shocks sobre la tasa nominal de interés aunque este efecto solo dura un trimestre. Shocks sobre el resto de las variables endógenas del sistema no tienen impacto sobre la tasa de inflación.

Los resultados también muestran que shocks positivos sobre la tasa de interés nominal tienen un impacto de igual signo positivo sobre la depreciación nominal. Una explicación posible a este hallazgo podría ser que expectativas crecientes de inflación inducirían a una fuga de la moneda doméstica al dólar, y a una consecuente depreciación nominal del peso. Este resultado contradice lo que habitualmente es encontrado en la literatura pero coincide con lo hallado para Argentina por Basco, D'Amato and Garegnani (2009) para el período de alta inflación 1977-1988. En cuanto al producto, se observa una respuesta negativa a shocks positivos sobre la tasa de interés aunque este efecto es solo significativo por un trimestre.

Asimismo, la respuesta del producto a los impulsos monetarios es positiva y significativa, perdurando por dos trimestres. Los shocks positivos sobre el tipo de cambio no tienen un impacto significativo sobre el resto de las variables endógenas con excepción del producto para el primer trimestre.

Por último, el crecimiento monetario responde negativamente a impulsos positivos en la tasa de interés, este impacto es poco persistente perdurando dos trimestres.

La tabla (11) muestra la descomposición de la varianza de la inflación que es nuestra variable de interés, los resultados indican que la inflación explica su propia variabilidad en un $50 \%$, siendo el crecimiento monetario la variable que, después de su propia dinámica, más 
explica su variabilidad ( $25 \%$ a partir del sexto trimestre), seguida por la devaluación nominal (11\% a partir del sexto trimestre).

Gráfico (6). Funciones de Impulso-Respuesta 2003-2013

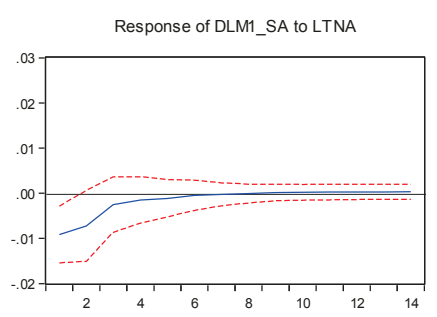

Response to Cholesky One S.D. Innovations \pm 2 S.E.
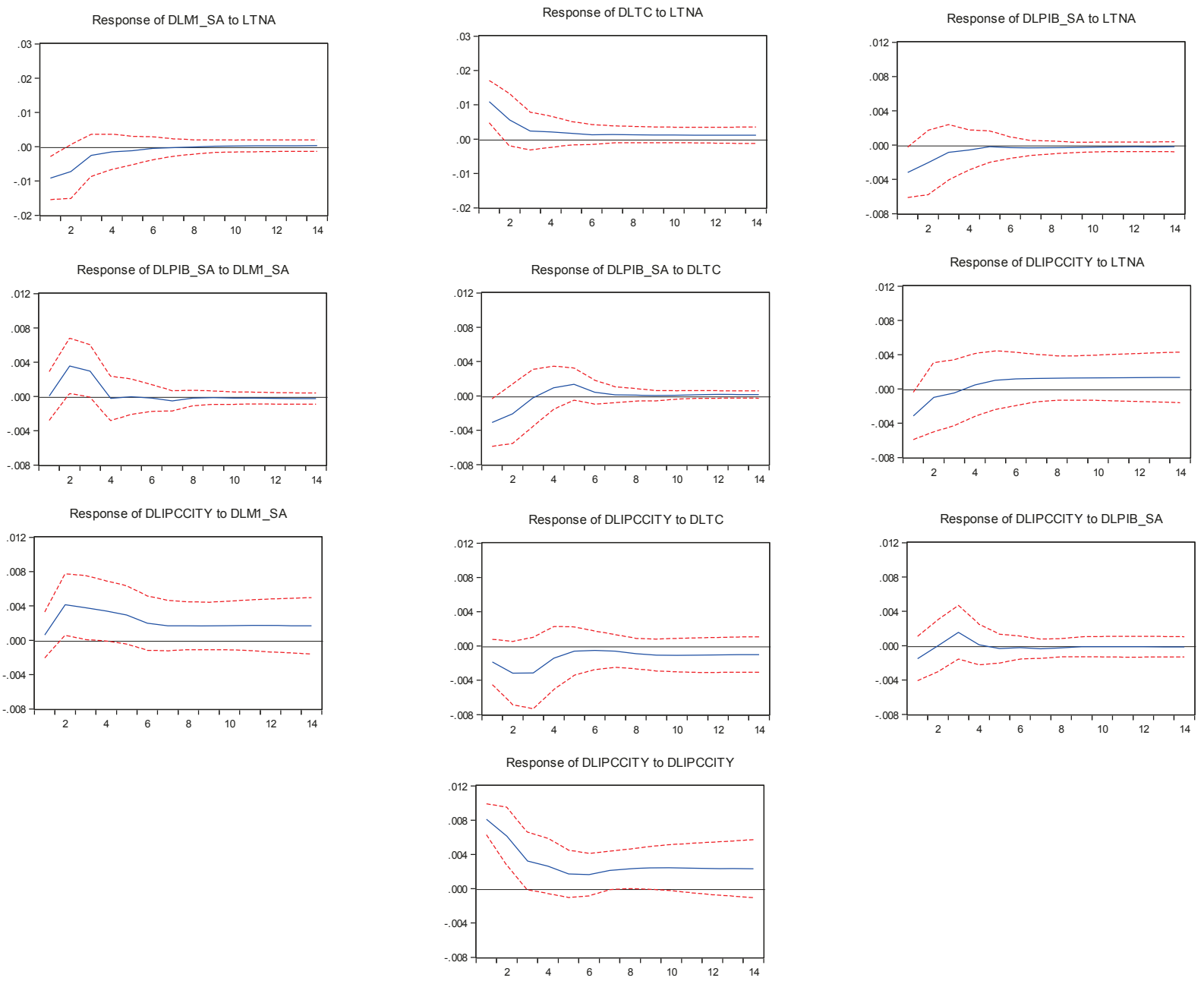

Tabla (11). Descomposición de Varianzas de la Inflación

\begin{tabular}{|c|c|c|c|c|c|c|}
\hline Trimestre & S.E. & LTNA & DLM1_SA & DLTC & DLPIB_SA & DLIPCCITY \\
\hline 1 & 0,009036 & 12,05752 & 0,478250 & 4,246149 & 2,753665 & 80,46441 \\
\hline 2 & 0,012138 & 7,325880 & 11,91596 & 9,186156 & 1,525904 & 70,04610 \\
\hline 3 & 0,013595 & 5,952192 & 17,30591 & 12,69822 & 2,530830 & 61,51284 \\
\hline 4 & 0,014341 & 5,456208 & 21,20421 & 12,38819 & 2,280631 & 58,67076 \\
\hline 5 & 0,014793 & 5,602748 & 23,88400 & 11,81163 & 2,195951 & 56,50567 \\
\hline 6 & 0,015071 & 5,999204 & 24,74429 & 11,49779 & 2,138201 & 55,62051 \\
\hline 7 & 0,015384 & 6,434522 & 24,96653 & 11,18310 & 2,109776 & 55,30607 \\
\hline 8 & 0,015728 & 6,807989 & 25,03500 & 11,02076 & 2,043728 & 55,09252 \\
\hline 9 & 0,016085 & 7,126785 & 25,00109 & 10,97751 & 1,960219 & 54,93439 \\
\hline 10 & 0,016449 & 7,432452 & 24,99717 & 10,93261 & 1,880712 & 54,75706 \\
\hline
\end{tabular}


En resumen, durante el período 2003-2013 se encuentra que los impulsos monetarios tienen un impacto positivo y significativo sobre la tasa de inflación mientras que la tasa de interés tiene un efecto negativo muy corto y poco significativo sobre la misma. Asimismo es importante resaltar que dos de los resultados encontrados en las funciones de impulso respuesta coinciden con resultados encontrados por Basco, D’Amato and Garegnani (2009) para el período considerado como de alta inflación. La descomposición de varianzas confirma la relevancia de los impulsos monetarios para explicar la volatilidad de la inflación

\section{Conclusiones}

En el trabajo se analizó la dinámica de la inflación agregada en el periodo 1980-2013, en particular el fenómeno de la persistencia inflacionaria. Para este análisis se utilizaron métodos recursivos y tests de cambio estructural desarrollados por Bai-Perron identificando quiebres en la tasa media de la inflación que coinciden con cambio de regímenes. Los más destacados son: el quiebre de agosto de 1989, el cual está en el entorno del primer episodio hiperinflacionario; y el quiebre a partir de noviembre de 2006. A partir del 2007 la inflación comienza a incrementase nuevamente debido al alto gasto público y aumento en el tipo de cambio real. Dada la presencia de cambios en la tasa media de inflación, diferenciamos esta variable respecto de esa media que evoluciona según quiebres discretos y calculamos medidas de persistencia inflacionaria.

Se encuentra que la inflación fue un proceso altamente persistente en el periodo de alta inflación y se redujo con el descenso de la misma, periodo de convertibilidad. Luego de la introducción del régimen de flotación administrada y controlando por el episodio inflacionario desatado por la devaluación del peso en 2002, encontramos que la inflación vuelve a mostrar un comportamiento más persistente. En el periodo 2003-2013, el cual se incluye una tendencia positiva, aumenta no solo la media si no la persistencia de la inflación, este incremento en el nivel de precios se debe al aumento en el gasto público y políticas que buscan expandir la demanda agregada, generando esto un aumento en la emisión monetaria.

Los resultados del análisis de persistencia corroboran la importancia de evaluar la presencia de quiebres estructurales al modelar la dinámica inflacionaria. También se confirma que en Argentina la persistencia no es una característica intrínseca de la inflación, si no dependiente del régimen monetario.

Además, luego de estudiar la persistencia de la inflación de manera univariada se procede a introducirla en un contexto multivariado a través de modelos VAR para comprender el 
vínculo de su dinámica aquellas variables económicas relevantes en la transmisión de los impulsos nominales a la economía.

En el periodo 2003-2013, se encuentra que shocks sobre la tasa de crecimiento del M1 tienen un impacto positivo sobre la inflación, esto afirma la ecuación cuantitativa del dinero la que dice que un aumento en la cantidad de dinero, origina un aumento en el nivel de precios.

Estos resultados sugieren que, el análisis multivariado permite una mejor comprensión de la dinámica de la inflación bajo distintos regímenes inflacionarios. 


\section{Bibliografía}

Altissimo, F., Bilke, L., Levin, A., Mathä, T., \& Mojon, B. (2006). Sectoral and Aggregate Inflation Dynamics in the Euro Area. Journal of the European Economic Association, Vol. 4, No. 2/3, 585-593.

Andrews, D., \& Chen, H. (1994). Approximately median-unbiased estimation of autoregressive models. Journal of Business \& Economic Statistics 12(2), 187-204.

Argandoña, A., Gámez, C., \& Mochón, F. (1997). Macroeconomía Avanzada. Tomo I. Madrid: McGraw Hill.

Bai, J., \& Perron, P. (2003). Computation and Analysis of Multiple Structural Change Models. Journal of Applied Econometrics, 18, 1, 1-22.

Basco, E., D'Amato, L., \& Garegnani, L. (2009). Understanding the money-prices relationship under low and high inflation regimes: Argentina 1977-2006. Journal of International Money and Finance. Nro 28, 1182-1203.

Benati, L. (2007). Drift and Breaks in Labor Productivity. European Central Bank. Working paper series, Nro 718.

Capistrán, C., \& Ramos-Francia, M. (2006). Inflation Dynamics in Latin America. Banco de México. Working Papers, Nro 11.

Cogley, T., \& Sbordone, A. (2006). Trend Inflation and Inflation Persistence in the New Keynesian Phillips Curve. Federal Reserve Bank of New York Staff Reports, Nro 270.

Cogley, T., Primiceri, G., \& Sargent, T. (2008). Inflation - GAP persistence en the U.S. National Bureau of Economic Research, Working Paper 13749.

D'Amato, L., \& Garegnani, L. (2013). ¿Cuán persistente es la inflación?: Regímenes inlfacionarios y dinamica de precios en los últimos 50 años. En L. D'Amato, E. López Enciso, \& M. Ramírez G., Dinámica inflacionaria, persistencia y formación de precios y salarios (págs. 91-115). México D. F.: CEMLA.

D'Amato, L., Garegnani, L., \& Sotes, J. (2008). Dinámica inflacionaria y persistencia: Implicancias para la política monetaria. Ensayos Económicos de BCRA, Nro 50, pag. 127.

Doepke, M., Lehnert, A., \& Sellgren, A. (1999). Macroeconomics. Chicago: University of Chicago. 
Ferrer, A. (2008). La Economía Argentina: desde sus orígenes hasta principios del siglo XXI. Argentina: Fondo de Cultura Económica.

Fuhrer, J. (2009). Inflation Persistence. Federal Reserve. Bank of Boston, Nro 09-14.

Fuhrer, J., \& Moore, G. (1995). Inflation Persistence. The Quarterly Journal of Economics, Vol. 110 , No. 1, 127-159.

Hamilton, J. (1994). Time Series Analysis. New Jersey: Princenton University Press.

Levin, A., \& Piger, J. (2004). Is inflation persistence intrinsic in industrial economies? European Central Bank. Working paper series, Nro 334.

Marques, C. (2004). Inflation Persistence: Facts or Artefacts. European Central Bank. Working paper series., Nro 371.

Sbordone, A. (2007). Inflation Persistence: Alternative Interpretations and Policy Implications. Federal Reserve Bank of New York Staff Reports, Nro 286.

Sims, C. (1980). Macroeconomics and Reality. Econometrica, Vol. 48, No. 1, 1-48.

Stock, J., \& Watson, M. (2006). Why Has U.S. Inflation Become Harder to Forecast? NBER Working Paper, Nro 12324. 


\section{Apéndices}

\section{Apéndice 1: Definiciones y fuentes de los datos}

En este apéndice describimos las series macroeconomías utilizadas en el trabajo.

\section{Tasa de Inflación}

La tasa de inflación es calculada como la variación porcentual del IPC de un periodo con respecto al periodo anterior:

$$
\text { Tasa de inflacón }=\frac{\left(I P C_{1}-I P C_{0}\right)}{I P C_{0}}
$$

Las fuentes para construir la serie son: INDEC, Buenos Aires City e IPC Congreso, Partiendo de que las tres series comparten la misma metodología de cálculo, se empalman obteniendo una serie homogénea desde 1980 a 2013,

El siguiente cuadro muestra los periodos y las fuentes de la serie,

\begin{tabular}{|l|l|}
\hline Periodo & Fuente \\
\hline $1980-2005$ & INDEC \\
\hline $2006-2010$ & Buenos Aires City \\
\hline $2011-2013$ & IPC Congreso \\
\hline
\end{tabular}

Para el análisis de persistencia se utiliza la serie mensual de inflación. Para el análisis VAR se utiliza la serie trimestral sacando promedios del nivel general del IPC y luego calculando la variación porcentual de los trimestres,

\section{Tasa de Interés Nominal Anual (TNA)}

Esta variable es obtenida del BCRA y es la tasa de interés por depósitos entre 30 y 59 días. Para el análisis VAR se utiliza esta serie sacando promedios de la tasa en tres meses y luego se calcula el logaritmo natural de 1+TNA,

\section{Tipo de Cambio}

Esta variable es obtenida del BCRA y se calcula en base al Tipo de Cambio Multilateral. El tipo de cambio utilizado es el tipo de cambio de Argentina con los tres principales socios comerciales (Brasil, Unión Europea y EEUU). Para el análisis VAR se utiliza esta serie sacando promedios en tres meses y luego se calcula el logaritmo natural, 


\section{Cantidad de Dinero (M1)}

Esta variable es obtenida del BCRA y es el circulante más los depósitos en cuenta corriente del sector privado. Para el análisis VAR se utiliza esta serie sacando promedios en tres meses, luego se calcula el logaritmo natural y se desestacionaliza con la función X12 del Eviews, con método de ajuste multiplicativo,

\section{Producto Bruto Interno}

Esta variable es obtenida del MECON y es el producto en pesos a precios de 1993. Para el análisis VAR se calcula el logaritmo natural y se desestacionaliza con la función X12 del Eviews, con método de ajuste multiplicativo,

\section{Apéndice 2: Descripción de periodos}

En este apéndice se describe cada periodo utilizado en el análisis del trabajo:

- 1980:1-1989:3: periodo de alta inflación,

- 1989:4-1990:3: episodio hiperinflacionario,

- 1990:4-1991:2: periodo de transición,

- 1991:3-1992:12: período de desinflación,

- 1993:1-2007:2: implementación de la convertibilidad y devaluación, acá termina el periodo estudiado por D'Amato, Garegnani, \& Sotes (2008),

- 2002:1-2002:9: crisis devaluación del peso,

- 2002:10-2007:2: devaluación del peso, salida de convertibilidad, adopción de un régimen de flotación administrada en 2002 ,

- 1980:1-2007:2: periodo completo de trabajo de D'Amato, Garegnani, \& Sotes (2008),

- 2007:1-2013:12: aporte de este trabajo,

- 1980:1-2013:12: periodo completo de este trabajo.

Apéndice 3: Desarrollo de las funciones de impulso-respuesta y la descomposición de varianza,

La especificación general de un modelo $\operatorname{VAR}(p)$ está dada por la siguiente expresión:

$$
Y_{t}=c+\emptyset_{1} Y_{t-1}+\emptyset_{2} Y_{t-2}+\cdots+\emptyset_{p} Y_{t-p}+\varepsilon_{t}
$$

Donde $Y_{t}$ es un vector de variables objeto de predicción, $Y_{t-1}, Y_{t-2}, \ldots, Y_{t-p}$ son los vectores de rezagos de las variables del sistema, los coeficientes $\emptyset$ son matrices de coeficientes a estimar, y $\varepsilon$ es un vector de perturbaciones aleatorias que cumplen los siguientes supuestos:

$$
\begin{aligned}
E\left(\varepsilon_{t}\right) & =0 \quad(7) \\
E\left(\varepsilon_{t} \varepsilon_{\tau}\right) & =\left\{\begin{aligned}
\Omega_{N x N}, & \text { para } t=\tau \\
0, & \text { para } t \neq \tau
\end{aligned}\right.
\end{aligned}
$$

\section{Funciones impulso-respuesta}


En la práctica, las interpretación del sistema VAR se basa en ejercicios de simulación, uno de ellos son las funciones impulso-respuesta, las cuales permiten investigar que sucede con el sistema ante variaciones exógenas,

Si consideramos la representación $\operatorname{VMA}(\infty)$ de $\operatorname{VAR}(p)$ :

$$
\begin{gathered}
Y_{t}=\sum_{s=0}^{\infty} \Psi_{s} \varepsilon_{t-s} \\
\Psi_{s}=I_{m}, \varepsilon_{t} \sim R B M^{5} \\
\frac{\partial Y_{t}}{\partial \varepsilon_{t-s}}=\frac{\partial Y_{t+s}}{\partial \varepsilon_{t}}=\Psi_{s}
\end{gathered}
$$

Donde:

$\Psi_{s}(S)$ : representa el efecto de un incremento en una unidad en la innovación de la j-ésima variable en el momento $t$, sobre el valor de la i-ésima variable en el momento $t+s$, permaneciendo todas las restantes innovaciones constantes para todo el momento del tiempo,

$\Psi_{s}(S) / \sqrt{V\left(\varepsilon_{j t}\right)}$ : representa el efecto sobre $Y_{i, t+s}$ de cambiar $\varepsilon_{j t}$ en un desvío estándar: función de impulso-respuesta simple,

\section{Descomposición de varianza}

Otra simulación para interpretar el sistema VAR es la descomposición de la varianza, la cual es una idea similar a R2 para descomponer la variabilidad total de una variable del VAR en términos de las variabilidades exógenas de cada una de las variables del sistema,

Partimos de la representación $\operatorname{VMA}\left({ }^{\infty}\right)$ de $\operatorname{VAR}(p)$ :

$$
\begin{gathered}
Y_{t}=\sum_{s=0}^{\infty} \Psi_{s} \varepsilon_{t-s} \\
\Psi_{0}=I_{m}, \varepsilon_{t} \sim R B M
\end{gathered}
$$

Que implica que:

$$
\begin{aligned}
V\left(Y_{t}\right) & =\sum_{s=0}^{\infty} \Psi_{s} \Omega \Psi_{s}^{\prime} \\
& =\sum_{s=0}^{\infty} \Psi_{s} \mathrm{HDH}^{\prime} \Psi_{s}^{\prime}
\end{aligned}
$$

\footnotetext{
${ }^{5}$ Ruido Blanco Multivariado
} 


$$
\begin{aligned}
& =\sum_{s=0}^{\infty} \Psi_{s}\left\{\sum_{i=1}^{N} d_{i} h_{i} h_{i}^{\prime}\right\} \Psi_{s}^{\prime} \\
& =\sum_{i=1}^{N} d_{i}\left\{\sum_{s=0}^{\infty} \Psi_{s} h_{i} h_{i}^{\prime} \Psi_{s}^{\prime}\right\} \\
& =\sum_{i=1}^{N} V\left(\mu_{i}\right)\left\{\sum_{s=0}^{\infty} \Psi_{s} h_{i} h_{i}^{\prime} \Psi_{s}^{\prime}\right\} \\
& =\sum_{i=1}^{N} V\left(\mu_{i}\right) B^{(i)}
\end{aligned}
$$

En particular la j-ésima varianza:

$$
\begin{gathered}
V\left(Y_{t j}\right)=\sum_{i=1}^{N} V\left(\mu_{i}\right) B_{j j}^{(i)} \\
1=\sum_{i=1}^{N} \frac{V\left(\mu_{i}\right) B_{j j}^{(i)}}{V\left(Y_{t j}\right)}=\sum_{i=1}^{N} z_{j i}
\end{gathered}
$$

Donde $z_{j i}=$ proporción de la varianza de la j-ésima variable que es explicada por el componente exógeno de cada una de las $\mathrm{i}=1,2, \ldots, \mathrm{N}$ variables del sistema,

\section{Apéndice 4: Análisis descriptivo y gráficos de variables del sistema VAR}

Siguiendo el mismo análisis que en el apartado anterior de persistencia, calculamos los estadísticos descriptivos de las series en estudio,

\begin{tabular}{|c|c|c|}
\hline & Periodo & $\mathbf{2 0 0 3 : 1 - 2 0 1 3 : 1 2}$ \\
\hline \multirow{2}{*}{ Inflación } & Media & 0,040 \\
\cline { 2 - 3 } & Desvío & 0,019 \\
\hline \multirow{2}{*}{ M1 } & Media & $133,777,53$ \\
\cline { 2 - 3 } & Desvío & $93,769,97$ \\
\hline \multirow{2}{*}{ TNA } & Media & 0,09 \\
\cline { 2 - 3 } & Desvío & 0,04 \\
\hline \multirow{2}{*}{ TC } & Media & 3,63 \\
\cline { 2 - 3 } & Desvío & 0,81 \\
\hline \multirow{2}{*}{ PIB } & Media & $373,656,30$ \\
\cline { 2 - 3 } & Desvío & $77,389,21$ \\
\hline
\end{tabular}




\section{Representación gráfica de series}

Tasa de Inflación

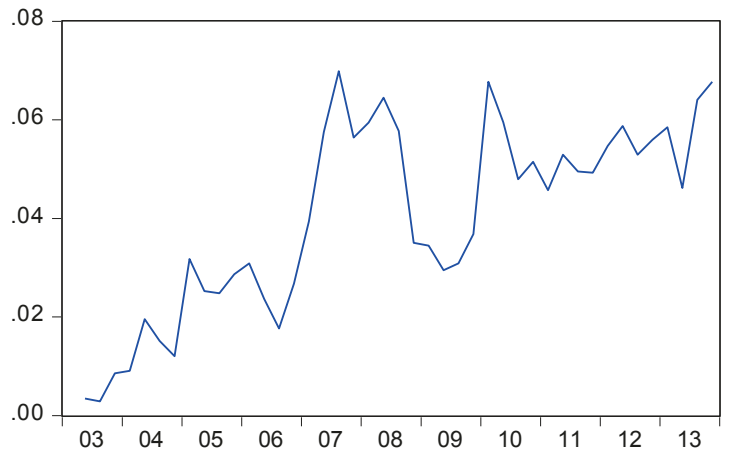

TNA

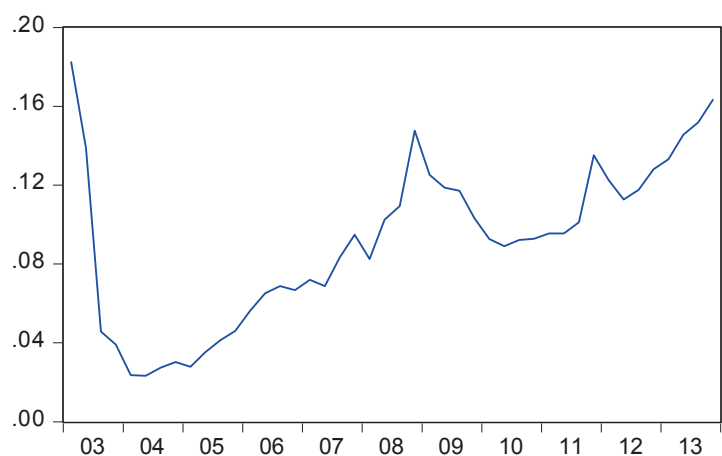

PIB

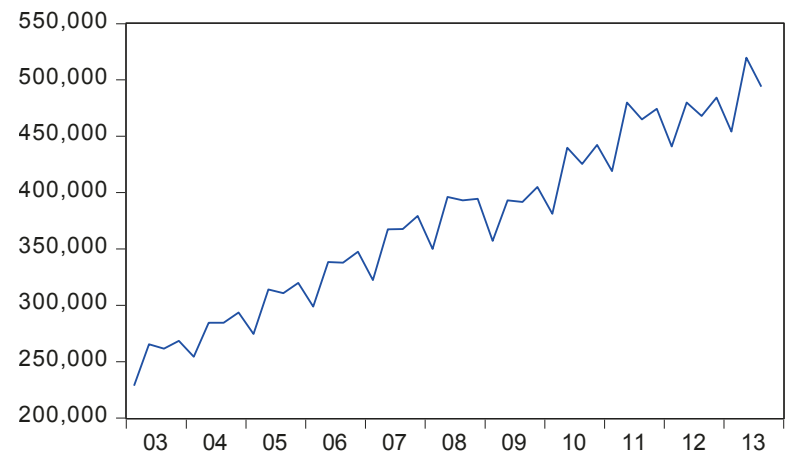

M1

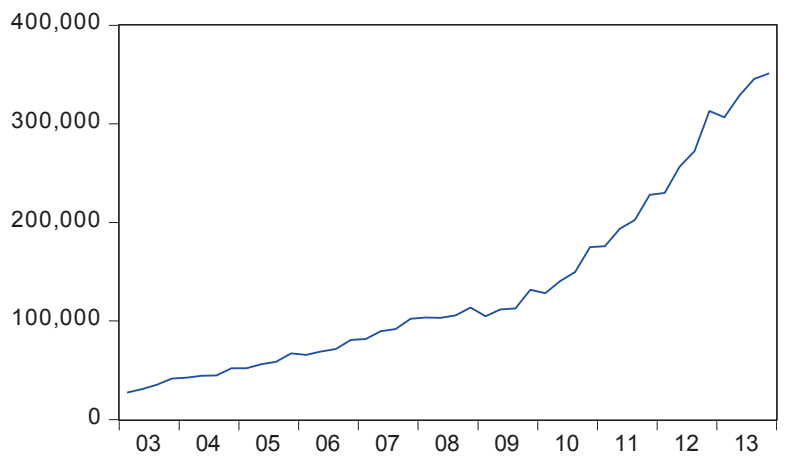

TC

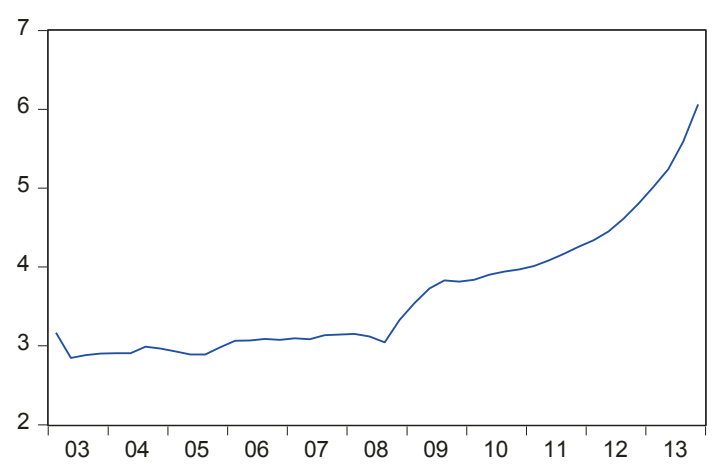

